Introduction/Background* Planning and consenting in major gynae-oncology surgery is a meticulous and important process. To maximise benefit to the patient it is imperative that the intent of treatment when planning major surgery is precise and specific. Additionally, to uphold informed consent and fulfil patient expectations, individuals must be accurately informed of the procedure they will undergo - a sentiment reflected in local, national, and international guidelines.

Methodology A quality Improvement project was conducted at a UK tertiary centre (New Cross Hospital, Wolverhampton) that aimed to evaluate the intent of treatment in major gynae-oncology surgery. 48 patients who underwent major procedures between October and December 2020 were selected for analysis. The primary objective was to ascertain the concordance between pre-operative planning and the actual procedure performed - this was determined by comparing the pre-operative documents with operation notes, and thereby expressing the concordance between procedures in the two as a percentage. Reasons for discrepancies found between pre-operative plans and the operations were then established. Other aspects evaluated included who examined patients (and when they were examined), how imaging was considered in pre-op decisions, and continuity of care throughout the surgical process.

Result(s)* It was found that overall concordance was $86.6 \%$ most patients underwent procedure in the manner that had been planned and expected. Reasons for discrepancy (i.e., the 13.4\% discordance) was categorised into 4 main categories: 1. Procedure not tailored to findings of imaging; 2. Unexpected intra-op findings; 3. Multiple surgical approaches consented; and 4. changes to surgical approach in vulvectomy. $89.4 \%$ of patients were examined in the pre-operative assessment - of

\begin{tabular}{|c|c|c|}
\hline $\begin{array}{l}\text { Concordance by Procedure } \\
\text { (operation plan vs operation } \\
\text { notes) }\end{array}$ & $\begin{array}{l}\text { Concordance of } \\
\text { procedures }\end{array}$ & $\%$ \\
\hline Laparoscopy & $9 / 15$ & $60.0 \%$ \\
\hline Laparotomy & $25 / 29$ & $86.2 \%$ \\
\hline Robotic & $5 / 12$ & $41.7 \%$ \\
\hline Hysterectomy & $31 / 31$ & $100.0 \%$ \\
\hline L salpingectomy & $34 / 35$ & $97.1 \%$ \\
\hline$R$ salpingectomy & $34 / 34$ & $100.0 \%$ \\
\hline L oophorectomy & $32 / 33$ & $96.9 \%$ \\
\hline R oophorectomy & $33 / 33$ & $100.0 \%$ \\
\hline Omentectomy & $7 / 11$ & $63.6 \%$ \\
\hline Lymphadenectomy & $10 / 17$ & $58.8 \%$ \\
\hline Vulvectomy & $8 / 8$ & $100.0 \%$ \\
\hline $\begin{array}{l}\text { Additional Procedures (including } \\
\text { biopsies) }\end{array}$ & $13 / 24$ & $56.5 \%$ \\
\hline TOTAL & $241 / 282$ & $85.5 \%$ \\
\hline
\end{tabular}

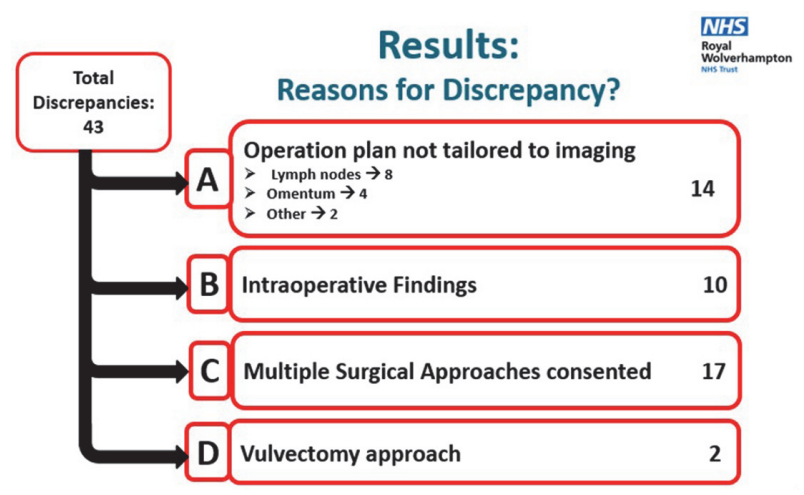

Abstract 993 Figure 1

those, $97 \%$ within 4 months of the operation. $76 \%$ of patients were operated on by the examining consultant, although this was found not to affect concordance.

Conclusion* Departmental recommendations were established from this Quality Improvement Project - The importance of considering imaging pre-operative decisions was emphasised, as well as recommendations to tailor consent to individuals in gynae-oncology surgery. Further recommendations included encouragement of sufficiently defining intended treatments in consent documentation, and promoting continuity/consistency of consultant in surgical care.

\section{IS THERE A ROLE FOR A MULTIDISCIPLINARY TUMOR BOARD SMART VIRTUAL ASSISTANT IN LOCALLY ADVANCED CERVICAL CARCINOMA? A PROOF OF CONCEPT}

${ }^{1} \mathrm{G}$ Macchia, ${ }^{2} \mathrm{G}$ Ferrandina, ${ }^{3} \mathrm{~S}$ Patarnello, ${ }^{4} \mathrm{R}$ Autorino* ${ }^{3} \mathrm{C}$ Masciocchi, ${ }^{3} \mathrm{~V}$ Pisapia, ${ }^{3} \mathrm{C}$ Calvani, ${ }^{3} \mathrm{C}$ lacomini, ${ }^{5} \mathrm{~A}$ Cesario, ${ }^{6} \mathrm{~B}$ Gui, ${ }^{7} \mathrm{~V}$ Rufini, ${ }^{4} \mathrm{~L}$ Boldrini, ${ }^{4} \mathrm{MA}$ Gambacorta, ${ }^{2} \mathrm{G}$ Scambia, ${ }^{4} \mathrm{~V}$ Valentini. ${ }^{1}$ Gemelli Molise Hospital - Università Cattolica del Sacro Cuore, Radiation Oncology Unit, Campobasso, Italy; ${ }^{2}$ Fondazione Policlinico Universitario A. Gemelli, IRCCS, UOC Ginecologia Oncologica, Dipartimento per la salute della Donna e del Bambino e della Salute Pubblica, Roma, Italy; ${ }^{3}$ Fondazione Policlinico Universitario A. Gemelli IRCCS, Rome, Italy; ${ }^{4}$ Fondazione Policlinico Universitario A. Gemelli IRCCS, UOC Radioterapia Oncologica, Dipartimento di Diagnostica per Immagini, Radioterapia Oncologica ed Ematologia, Roma, Italy; ${ }^{5}$ Fondazione Policlinico Universitario A. Gemelli IRCCS, Scientific Directorate, , Rome, Italy; ${ }^{6}$ Fondazione Policlinico Universitario A. Gemelli, IRCCS., UOC Radiologia, Dipartimento di Diagnostica per Immagini, Radioterapia Oncologica ed Ematologia, , Roma, Italy; ${ }^{7}$ Fondazione Policlinico Universitario A. Gemelli, IRCCS, UOC Medicina Nucleare, Dipartimento di Diagnostica per Immagini, Radioterapia Oncologica ed Ematologia, Roma, Italy

\subsection{6/ijgc-2021-ESG0.325}

Introduction/Background* The first prototype of the "Multidisciplinary Tumor Board Smart Virtual Assistant" is presented.

Methodology Data from patients affected by invasive carcinoma of the cervix (LACC), FIGO stage IB2-IVa, treated between 2015 and 2018 were extracted. Magnetic Resonance (MR), Gynecologic examination under general anesthesia (EAU), and Positron Emission Tomography-Computed Tomography (PET-CT) performed at the time of diagnosis were the items from the Electronic Health Records (eHRs) considered for analysis. An automated extraction of eHR that capture the patient's data before the diagnosis and then, through Natural Language Processing (NLP), analysis and categorization of all data to transform source information into structured data has been performed. Thereafter, an Artificial 


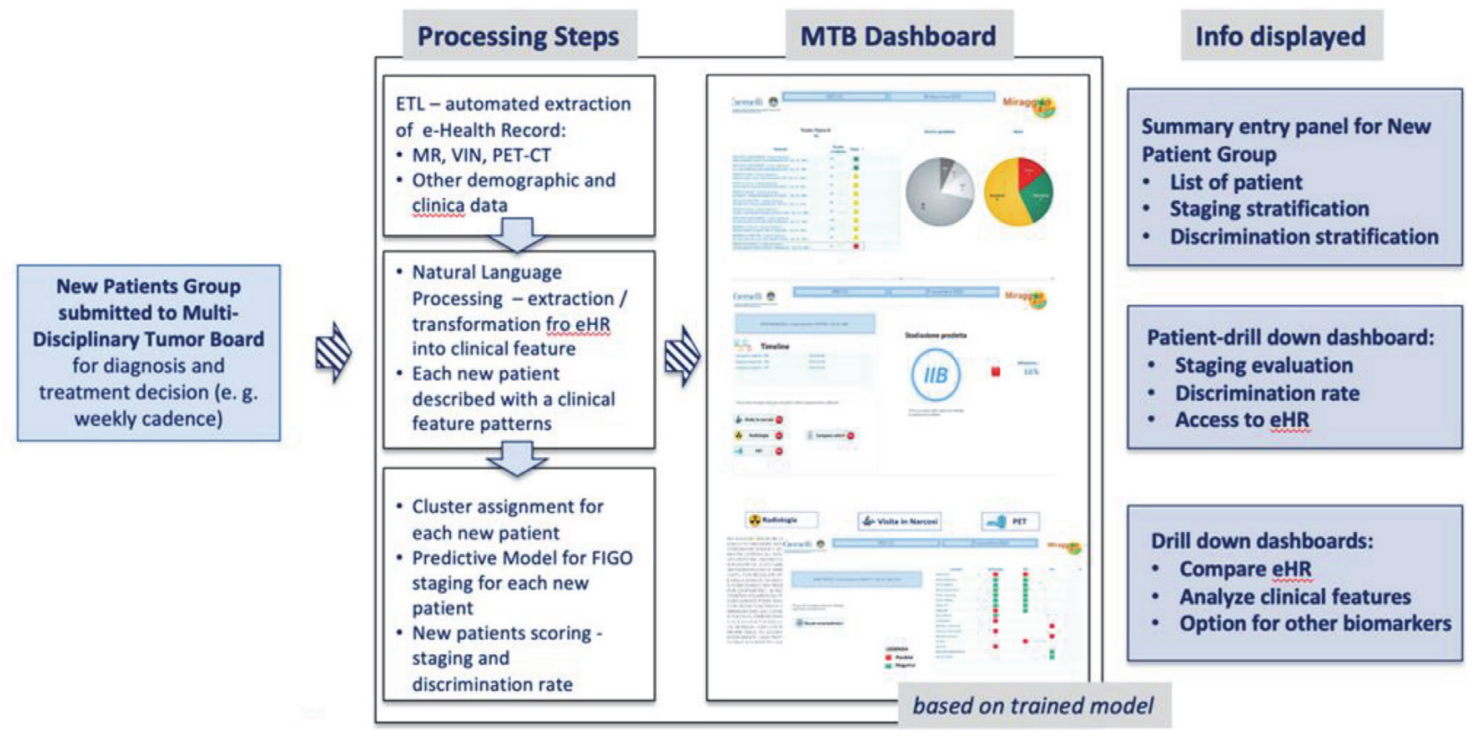

\section{Abstract 1014 Figure 1}

Intelligence method was developed to support the clinical staff in their decision with regards to tumor staging and to help them identifying the most complex cases where deeper analysis and discussion were required (e. g. conflicting information from different exams).

Result(s)* In the first round, the system has been used to retrieve all the eHR for the 96 patients with LACC. This was the training set of the study, with validated 2009 FIGO staging classification ranging from I B2 to IV A as output. For these patients, available eHR included MR, EUA, and PET-CT diagnostic reports. The system has been able to classify all patients belonging to the training set and - through the NLP procedures - the clinical features were analyzed and classified for each patient. A second important result was the setup of a predictive model to evaluate the patient's staging. Our approach has led to predict patient's staging within an accuracy of 94\%. Lastly we created a user-oriented operational tool targeting the MTB who are confronted with the challenge of large volumes of patients to be diagnosed in the most accurate way. The resulting decision support system is summarized in figure 1. Furthermore, the MTB Smart DA was tested in a 13 LACC patients validation cohort showing an accuracy of $93 \%$, in line with the training set performances.

Conclusion* This is the first proof of concept concerning the possibility of creating a smart virtual assistant for the MTB. A significant benefit could come from the integration of these automated methods in the collaborative, crucial decision stages.

\section{IMPACT OF COVID 19 ON ENDOMETRIAL CANCER FOLLOW-UP STRATEGIES}

${ }^{1}$ A Amirthanayagam*, ${ }^{2} \mathrm{~L}$ Boulter, ${ }^{1} \mathrm{H}$ Morris, ${ }^{3} \mathrm{~T}$ Miles, ${ }^{4} \mathrm{H}$ Manderville, ${ }^{1} \mathrm{E}$ Moss. ${ }^{1}$ University of Leicester, UK; ${ }^{2}$ Leicester Royal Infirmary, UK; ${ }^{3}$ Royal United Hospital, Bath, UK; ${ }^{4}$ Queen Elizabeth Hospital Gateshead, UK

10.1136/ijgc-2021-ESG0.326
Introduction/Background* The COVID-19 pandemic has had a dramatic impact on many aspects of healthcare, with changes in clinical practice aimed at reducing hospital appointments for patients who are classified as clinically vulnerable. In oncology this has resulted in a move towards the uptake of innovative follow-up schemes, such as patient-initiated or telephone follow-up, in particular for patients who are deemed to be at lower risk of recurrence. The aim of this study was to explore clinician's views of the impact of the COVID19 pandemic on the uptake of such schemes and the challenges in maintaining such schemes in the future.

Methodology A mixed methods study was performed consisting of an online questionnaire of clinical nurse specialists and a qualitative interview study of clinicians involved in the follow-up of patients after a diagnosis of endometrial cancer. The interviews were conducted virtually, audio recorded, transcribed verbatim and analysed using framework analysis.

Result(s)* In total, 22 clinical nurse specialists responded to the survey. Interview participants included gynaecological oncologists, cancer unit gynaecologist, oncologists and clinical nurse specialists. There was universal support to reduce faceto-face consultations during the COVID-19 pandemic and strong support for telephone and patient initiated follow-up. $70 \%$ of the participants of the online survey reported that in their opinion COVID-19 pandemic had had an impact on their clinic service by causing accelerated transition to patient initiated or telephone follow-up. The participants also reported patient support for the introduction of virtual follow-up compared to face-to-face appointments.

Conclusion* The COVID-19 pandemic has resulted in a transition towards telephone and patient initiated follow-up for the follow-up of endometrial cancer. Further research is needed to ensure that these schemes are able to address patients' clinical and information needs if the change from face-to-face clinical follow-up is to be continued following the COVID-19 pandemic. 